\title{
Fiscalidad de la economía digital
}

\section{Javier EnRIQue Reyes Sarmiento}

Abogado por la Universidad Nacional Mayor de San Marcos. Egresado de la Maestría en Política y Administración Tributaria de la

Universidad Nacional Mayor de San Marcos.

Post Título en Derecho Tributario por la Pontificia

Universidad Católica del Perú.

Diplomado en Tributación por el Centro Interamericano de

Administraciones Tributarias.

SUMARIO:

I. La economía digital.

II. Fiscalidad directa de las rentas obtenidas por la EMNT.

1. Enfoque multilateral: modelo $0 C D E$.

2. Enfoque multilateral: Modelo Unión Europea — UE.

2.1. Impuesto a los Servicios Digitales - ISD.

2.2. Presencia Digital Significativa.

3. Respuesta unilateral de varios países. 


\section{RESUMEN:}

La globalización de la economía, el desarrollo de la tecnología y la proliferación de servicios digitales ha generado la aparición de un nuevo orden fiscal internacional, ello ante la insuficiencia de los criterios de sujeción tradicionales — fuente y residencia — con la finalidad de gravar esta fuente de riqueza.

Es en este contexto, con una acentuada necesidad de recursos de todas las naciones, que hoy se discute en el mundo la necesidad de un nuevo nexo para la economía digital; no obstante, las propuestas actuales se desarrollan en torno a la posibilidad de los países desarrollados de gravar tal fuente, que pese a ser países de la residencia de las empresas prestadoras de estos servicios digitales, hoy se identifican, además como países de la fuente de esta fuente de riqueza.

En el presente trabajo, el autor analiza el contexto actual de la tributación de las empresas digitales no domiciliadas, abordando el mismo desde un punto de vista histórico, y luego observa su desarrollo legal a nivel internacional.

Palabras clave: fiscalidad global, economía digital, servicios digitales, establecimiento permanente, empresas multinacionales.

\footnotetext{
ABSTRACT:

The globalization of the economy, the development of technology and the proliferation of digital services has generated the appearance of a new international tax order, this given the insufficiency of the traditional subjection criteria — source and residence — with the purpose of tax this source of wealth.

It is in this context, with an accentuated need for resources from all nations, that today the world is discussing the need for a new nexus for the digital economy; however, the current proposals are developed around the possibility of developed countries to tax such a source, which despite being countries of residence of the companies that provide these digital services, today are also identified as countries of the source of this source of wealth.

In the present work, the author analyzes the current context of the taxation of non-domiciled digital companies, addressing it from a historical point of view, and then observe its legal development at the international level.

Keywords: global taxation, digital economy, digital services, permanent establishment, multinational companies.
}

\section{LA ECONOMÍA DIGITAL}

Desde los inicios de la civilización, las actividades económicas más importantes se encontraban relacionadas con la subsistencia -la agricultura, la ganadería, la manufactura y el comercio-, hasta que durante los siglos XVIII y $X I X$ se inventaron las maquinas a vapor y luego a motor durante la revolución industrial iniciada en Inglaterra, que sentó las bases de nuestro modo de vida moderno.

Sin embargo, a partir del siglo $\mathrm{XX}$ se dieron grandes avances en la electrónica en general, los superordenadores y el internet, lo cual generó las tecnologías de la información y la comunicación - TIC, que proporcionó la cobertura para crear el núcleo de la economía di- gital en el siglo XXI, como fenómeno disruptivo - que establece un nuevo paradigma de la economía-, y transversal - que afecta a todos los ámbitos de la economía-, cuya revolución nos permite intercambiar información con cualquier persona conectada a internet, realizar procesos comerciales o de servicios, impulsado la innovación en todos los sectores de la economía.

Las Empresas Multinacionales Tecnológicas EMNT han sido los principales agentes de este proceso, las cuales han creado diversos modelos de negocios ofreciendo una serie de servicios digitales, desde sus servidores ubicados principalmente en territorio de los países tecnológicamente avanzados ${ }^{1}$ a millones de usuarios en casi todos los países del mundo, donde

1. Estados Unidos de América, China, Corea del Sur, Alemania, Finlandia, Suecia, por citar algunos ejemplos. 
no siempre tienen presencia física, ente los cuales cabe destacar los siguientes:

a) Comercio electrónico, como la venta de productos físicos o servicios a través de la red mediante plataformas específicas. Puede acontecer en entornos B2B - Business to Business-, B2C - Business to Consumer- o C2C - Consumer to Consumer-, en los que la empresa desarrolla el rol de intermediario y procediendo sus ingresos bien de los propios consumidores o usuarios o bien de servicios publicitarios de terceros empresarios. Incluye servicios de intermediación a través de plataformas que contactan a dos personas, tales como Airbnb en el arriendo de inmuebles; o contactan a empresas con usuarios, como las aplicaciones de transporte —Uber, Cabify, etc.-, o de entrega de bienes desde comercios establecidos al domicilio de los consumidores - como Rappi, UberEats, etc.- , compras en el extranjero o comercio digital a través de plataformas digitales —como Amazon, Aliexpress, eBay, etc-.

b) Servicios o plataformas de pagos, en las que el empresario digital actúa de intermediario entre comprador y vendedor.

c) Servicios de descarga de aplicaciones y productos digitales. Incluye las aplicaciones de entretenimiento y streaming pay per view contratadas por particulares de música o películas, tales como Spotify, Netflix, etc.; aplicaciones de telecomunicaciones -Zoom, Teams, etc.-, entre otros.

d) Publicidad Online.

e) Plataformas de red participativas, frecuentemente llamadas redes sociales, tales como Google, Facebook, Instagram, etc. En este caso, si bien las EMNT no les cobran a los usuarios por el uso de sus plataformas, el aprovechamiento se da por la publicidad y por la obtención de información de los usuarios, para efectos de ser vendida a terceros, con fines principalmente comerciales.

f) Cloud Computing: comprende la prestación de servicios digitales y estandarizados relativos a la programación informática, almacenamiento de datos en servidores ubicados fuera de la jurisdicción, gestión de software y datos y la utilización compartida de recursos físicos y virtuales.

g) Explotación de datos: La información obtenida de consumidores, usuarios y empresas a través de múltiples fuentes - como uso de buscadores y de redes socialesconstituye un activo intangible muy importante que es objeto de tratamiento de cara a su explotación comercial por empresas y organizaciones, frecuentemente en entornos internacionales. ${ }^{2}$

Si bien el vertiginoso crecimiento de la economía digital fue impulsado por políticas de no intervención de los Estados, en los últimos años constituye un tema de especial trascendencia en materia de fiscalidad internacional, pues ha trastocado la aplicación de los principios tradicionales de imposición —residencia, EP o nexos típicos de fuente-, por lo que surge la interrogante de establecer a qué país se debe asignar la potestad tributaria sobre los ingresos obtenidas por las EMNT que desarrollan este tipo de actividades en múltiples jurisdicciones, teniendo en cuenta que no hay presencia física de dichos proveedores en el lugar de consumo.

\section{FISCALIDAD DIRECTA A LAS RENTAS OB- TENIDAS POR LAS EMNT}

En el Perú, el Impuesto a la Renta grava las ganancias netas generadas por los sujetos domi-

2. Información obtenida de los usuarios, tal como: ¿qué compran?, ¿cómo pagan?, ¿en qué entidad financiera tienen su dinero?, ¿qué medio de pago utilizan?, etc., y que sirve como insumo para crear - a través de la inteligencia artificial - una base de datos global con una vasta información de tales usuarios, que permite la prestación de servicios de "Big Data". 
ciliados en el país, así como las rentas de fuente nacional obtenidas por los sujetos no domiciliados. Por tanto, las rentas que obtengan las EMNT no domiciliadas en el país por los servicios digitales que sean utilizados económicamente en el país, califican como rentas de fuente peruana gravadas con dicho impuesto y lo pagan a través de las empresas domiciliadas usuarias de los mismos ${ }^{3}$; sin embargo, cuando dichos servicios se prestan a personas naturales sin actividad empresarial, no están sujetas al pago de dicho impuesto.
Tradicionalmente, el criterio de conexión predominante del impuesto a la renta ha sido la presencia física, esto es, el "lugar físico de negocios", la residencia, la fábrica, el establecimiento comercial o similares, es decir, que los beneficios o ganancias de las empresas multinacionales sean gravados por el Estado en el cual éstas fijan su residencia, la cual generalmente está vinculada al lugar donde se encuentra su dirección efectiva.

Asimismo, se ha establecido la regla de la em-

3. De conformidad con el artículo 6 del Texto Único Ordenado de la Ley del Impuesto a la Renta aprobado por Decreto Supremo № 179-2004-EF, en caso de contribuyentes no domiciliados en el país, de sus sucursales, agencias o establecimientos permanentes, el impuesto recae solo sobre rentas gravadas de fuente peruana.

El inciso i) del artículo 9 de la citada ley, establece que en general y cualquiera sea la nacionalidad o domicilio de las partes que intervengan en las operaciones y el lugar de celebración o cumplimiento de los contratos, se considera rentas de fuente peruana las obtenidas por servicios digitales prestados a través del internet o de cualquier adaptación o aplicación de los protocolos, plataformas o de la tecnología utilizada por internet o cualquier otra red a través de la que se presten servicios equivalentes, cuando el servicio se utilice económicamente, use o consuma en el país.

El inciso j) del artículo 56 dispone que el impuesto a las personas jurídicas no domiciliadas en el país se determina aplicando la tasa del $30 \%$ a las otras rentas no mencionadas en los incisos anteriores, las cuales incluyen las rentas por servicios digitales.

El inciso c) del artículo 71 de la referida ley dispone que son agentes de retención las personas o entidades que paguen o acrediten rentas de cualquier naturaleza a beneficiarios no domiciliados.

El artículo 76 de la Ley, prescribe que las personas o entidades que paguen o acrediten a beneficiarios no domiciliados rentas de fuente peruana de cualquier naturaleza, deberán retener y abonar al fisco con carácter definitivo dentro de los plazos previstos por el Código Tributario para las obligaciones de periodicidad mensual, los impuestos a que se refiere el artículo 56 de la misma ley, entre otros.

Por su parte, el primer párrafo del inciso b) del artículo 4-A del Reglamento de la Ley del Impuesto a la Renta, dispone que se entiende por servicio digital a todo servicio que se pone a disposición del usuario a través del internet o de cualquier adaptación o aplicación de los protocolos, plataformas o de la tecnología utilizada por internet o cualquier otra red a través de la que se presten servicios equivalentes mediante accesos en línea y que se caracteriza por ser esencialmente automático y no ser viable en ausencia de la tecnología de la información; y que para efectos del referido reglamento, las referencias a página de internet, proveedor de internet, operador de internet o internet comprenden tanto a internet como a cualquier otra red, pública o privada.

El segundo párrafo del citado inciso b) señala que se consideran servicios digitales, entre otros, a los siguientes: (i) Mantenimiento de software; (ii) Soporte técnico al cliente en red; (iii) Almacenamiento de información —Data warehousing-; (iv) Aplicación de hospedaje —Application Hosting-; (v) Provisión de servicios de aplicación — Application Service Provider - ASP_; (vi) Almacenamiento de páginas de internet —website hosting —; (vii) Acceso electrónico a servicios de consultoría; (viii) Publicidad —-Banner ads—; (ix) Subastas "en línea"; (x) Reparto de Información; (xi) Acceso a una página de internet interactiva; (xii) Capacitación Interactiva; y (xiii) Portales en línea para compraventa.

El numeral 1 del tercer párrafo del citado inciso b) señala que el servicio digital se utiliza económicamente, se usa o se consume en el país, cuando sirve para el desarrollo de las actividades económicas de un contribuyente perceptor de rentas de tercera categoría domiciliado, con el propósito de generar ingresos gravados o no con el impuesto, siendo que se presume que un contribuyente perceptor de rentas de tercera categoría que considera como gasto o costo la contraprestación por el servicio digital, utiliza económicamente el servicio en el país.

De lo antes expuesto se desprende que para que un servicio sea considerado como digital, deberá cumplirse con los siguientes requisitos: (i) que sea un servicio; (ii) que se preste a través del internet o de cualquier adaptación o aplicación de los protocolos, plataformas o de la tecnología utilizada por internet o cualquier otra red a través de la que se presten servicios equivalentes; (iii) que se ponga a disposición mediante accesos en línea; (iv) que se caracterice por ser esencialmente automático; y (v) que no sea viable en ausencia de la tecnología de la información. 
presa separada, según la cual, en una empresa transnacional, cada empresa del Grupo, la Matriz y las Sucursales, son empresas separadas que tienen su tributación en el país donde tienen su Residencia. ${ }^{4}$ Sin embargo, la empresa matriz y la empresa separada tienen una única excepción a la regla anterior sobre tributación en el lugar de residencia, que es la existencia de un "Establecimiento Permanente" -EP-, que permite al país de la fuente gravar rentas empresariales generadas por residentes en otro Estado, pero si no se alcanza dicho status, no se tributa en el país de la fuente sino exclusivamente en el Estado de la residencia.

Para adquirir el status de EP, el artículo $5^{\circ}$ del Modelo de Convenio para evitar la Doble Imposición -2017- de la Organización de Cooperación y Desarrollo Económico - OCDE, en adelante, "MCOCDE", ha establecido que se deben cumplir los siguientes requisitos: a) Lugar fijo de negocios, entendido fundamentalmente como presencia física ${ }^{5}$; b) Si se tenía un agente -representante-, éste tenía que ser dependiente, es decir, con poderes para contratar en nombre y por cuenta de la Matriz, y c) Excepciones: no se considera como EP el realizar actividades auxiliares o preparatorias, como por ejemplo, solamente contar con almacenes -en el Estado de la Fuente- para la entrega de la mercaderías que comercializa.

Las reglas antes mencionadas han generado una suerte de inmunidad tributaria a los modelos de negocio que realizan las EMNT, pues en virtud al criterio de conexión predominante, tributan solamente en aquellos Estados donde han declarado o fijado su residencia o domicilio fiscal. Sin embargo se advierte que no tributan en los Estados donde obtienen sus ingresos: a) por la prestación de servicios digitales a personas naturales, o b) por la obtención y/o explotación de los datos obtenidos de los usuarios de sus plataformas ${ }^{6}$, por cuanto: 1) no tienen residencia o tienen una presencia física reducida en los Estados donde prestan servicios u obtienen los datos, y/o 2) las actividades realizadas en la jurisdicción de la fuente escapan de la definición de EP como lugar físico de negocios.

El problema se agrava cuando las EMNT operan a través de titulares de los intangibles explotados en la economía digital que fijan su domicilio en territorios o países de baja o nula imposición, conocidos como "paraísos fiscales".

Esta situación ha generado una preocupación a nivel internacional, toda vez que las bases fundamentales de la tributación a nivel internacional - conceptos como "el criterio de la fuente" o el "establecimiento permanente"no servirán para generar ingresos tributarios a los distintos estados en la economía digital. Países emergentes que no son miembros de la OCDE, tales como India, Brasil, México, los cuales tienen una enorme potencialidad para que las EMNT realicen sus negocios, pues son los países donde hay mayor volumen de población de consumidores, y son los que más interesados están en cambiar las reglas de localización de donde se van a gravar los beneficios por los servicios digitales, los que han solicitado que se establezca un impuesto unilateral sobre los servicios digitales; en cambio, los países donde la mayoría de EMNT tienen su residencia legal, han optado por no hacer nada.

A fin de gravar con un impuesto directo a los

4. Lo cual permite que existan erosiones de bases imponibles, así como traslados de beneficios de una jurisdicción a otra. Para evitar tales distorsiones, se estableció el régimen de precios de transferencia entre empresas vinculadas.

5. El concepto de lugar fijo de negocios deviene en obsoleto para la economía digital, dado que tiene su origen en los establecimientos de los comerciantes germanos de fines del siglo XVIII.

6. La información obtenida de los usuarios de sus plataformas permite elaborar un perfil del mismo, y se convierte en un bien intangible de propiedad de las EMNT, la cual puede comercializarse a terceros, obteniendo ganancias sin tributación alguna. 
beneficios de las EMNT dedicadas a la cada vez más creciente economía digital, que hacen negocios en países donde no tienen presencia física, se han formulado las siguientes propuestas fiscales:

\section{Enfoque multilateral: Modelo OCDE.}

La OCDE es un organismo de cooperación internacional conformado por los Estados con mejores economías del mundo, cuyo objetivo es coordinar sus políticas económicas y sociales, para lo cual, publica constantemente documentos de trabajo en diversas materias, entre las cuales cabe destacar la de fiscalidad internacional.

En octubre de 2005, la OCDE aprobó la denominada "Acción 1 del Proyecto BEPS"7 —2013-, en cuyo Informe Final ${ }^{8}$ propuso como solución internacional coordinada las siguientes recomendaciones: a) la modificación de la lista de excepciones a la definición de EP, para que se excluyan a actividades previamente calificadas como preparatorias o auxiliares y que han tenido un impacto significativo en los modelos de negocio digitales, como por ejemplo el almacenamiento de productos que se venden online ${ }^{9}$; b) la modificación de la definición de EP para incluir aquellos casos en los que se utilizan estructuras artificiosas para la venta de bienes o servicios de una empresa de un grupo multinacional se traduce en la práctica, en la celebración de contratos, de modo que habrá que considerar que esas ventas han sido efectuadas por dicha empresa; c) establecer que la titularidad jurídica de los intangibles no genera, por sí sola, el derecho a percibir los beneficios de su explotación y el derecho a percibir los beneficios de su explotación.

Cabe destacar que en los Comentarios 122 a 131 del MCOCDE, se analizaron los conceptos del Sitio web y el Servidor, concluyéndose que no resultaban suficientes para calificar como EP. ${ }^{10}$

Ante tal situación, algunos países consideraron que la mencionada Acción 1 no cumplía el objetivo de gravar con impuesto directo los beneficios generados por los distintos servicios digitales, por lo que desde el año 2010, empezaron a formular como propuesta multilateral alternativa que se cambie el "nexo", es decir, que se sustituya la regla de residencia física de la empresa por la regla de "jurisdicción del mercado", como el territorio donde están ubicados los consumidores o usuarios, pues consideran que lo relevante en la economía digital no es

7. Denominada "Addressing the Tax Challenges of the Digital Economy" —los desafíos de la economía digital para la tributación internacional-.

8. Disponible en: https://www.oecd.org/ctp/beps-resumenes-informes-finales-2015.pdf

9. Ello debido a la proximidad con el consumidor y la necesidad de realizar una entrega rápida al cliente son dos factores que llevarían a concluir que el almacén local constituye un EP para el vendedor. Esta modificación supondría un importante cambio en el gravamen de multinacionales como Amazon, que tiene importantes centros de distribución en diferentes países y que, hasta el momento, no constituían un EP.

10. Según los aludidos comentarios, se señaló que el website — combinación de software y datos electrónicos- "no constituye un activo tangible como una maquinaria o un equipo informático, pues no tiene una presencia física que pueda considerarse como un lugar de negocios", por tanto, no constituye un EP.

En el caso del "Servidor" - en el que la website es almacenado—, se señaló que "para que sea considerado un lugar fijo de negocios, se requiere demostrar una conexión entre dicho lugar fijo de negocios y la actividad empresarial de la empresa, y que, a su vez, dicha actividad no sea calificada una actividad auxiliar o preparatoria. Asimismo, se deniega la posibilidad de considerar al servidor como un agente dependiente de la entidad no residente, pues en ningún caso concluye contratos y tampoco desarrolla el rol principal de la negociación de los mismos en nombre de la empresa, mientras que el proveedor de servicios de internet puede ser considerado como un agente independiente que actúa en el ámbito ordinario de su actividad y, además, presta servicios a distintas empresas". Al final, se recomienda examinar caso por caso. 
donde residen las EMNT sino donde se crea o genera el valor.

Por su parte, la OCDE formuló otras alternativas de carácter multilateral para la creación de normas autónomas de fiscalidad directa aplicables a la economía digital, aunque sin haber desarrollado directrices para su aplicación:

a) Presencia económica significativa que propone establecer un nuevo criterio de sujeción -nexo- en fuente que posibilite la existencia de un EP en modelos de negocio profundamente desmaterializados, como es la obtención y explotación de la información de los usuarios o consumidores de las plataformas, a fin de redistribuir las rentas del Estado de residencia de las EMNT a los distintos Estados de la fuente - donde se ubican los usuarios-. Esta figura reconoce la importancia del "Principio de Creación de Valor" de la "Jurisdicción del Mercado", vinculando la renta al lugar donde están los consumidores o usuarios de servicios, quienes generan los intangibles de comercialización.

Esta propuesta supone asignarle un valor a la información y datos obtenidos de manera remota - pese a que las EMNT no tienen una presencia física relevante en el territorio de la fuente-, los cuales pueden ser: visitas a páginas de internet, residencia de consumidores, búsquedas efectuadas en servidores, preferencias de consumo, compras digitales, transacciones financieras y pagos digitales o mediante tarjeta bancaria, etc.

No obstante, esta alternativa presenta dificultades en cuanto a que no se puede calcular de manera objetiva la creación de valor, toda vez que resulta difícil establecer: 1) la asignación de los beneficios obtenidos por las EMNT a cada jurisdicción, esto es, calcular el consolidado global de los beneficios antes de impuestos, así como repartir dichos beneficios en base a los importes de porcentaje del beneficio residual estimado, remuneración fija para fun- ciones de comercialización y distribución, y retorno adicional basado en el análisis de precios de transferencia; y 2) el acceso de los Estados de la fuente - donde se ubican los usuarios - a la información relacionada con el dominio de las plataformas de las EMNT, las transacciones o medios de pago financieros realizados en el país, el volumen de datos obtenidos en el territorio, el número de usuarios, la suscripción de contratos de modo online, entre otros datos relevantes.

b) Retención en la fuente para las operaciones digitales. El uso de retenciones en la fuente sobre el importe bruto es sencillo y puede utilizarse independientemente, sin el nexo de la presencia económica significativa, como en las retenciones en la fuente sobre las rentas obtenidas por una entidad no domiciliada que sean calificadas como dividendos, intereses o regalías. En la medida en que no se permite la deducción de gastos, es recomendable que la tasa sea reducida.

Esta alternativa implica que se designen a los sujetos que realizarán la retención, esto es, quiénes serían los denominados "collecting agents", en cuyo caso, no habría grandes inconvenientes en el caso de transacciones B2B; no obstante, en los supuestos de transacciones $B 2 C$, hay que tener en cuenta las dificultades que podría suponer para un consumidor final el hecho de tener que cumplir con esta obligación, por lo que resultaría aconsejable requerir para ello a los intermediarios en el procesamiento del pago, esto es, instituciones financieras implicadas en los pagos derivados de transacciones digitales, incluyendo a las compañías de tarjetas de crédito.

A fines del año 2019, la OCDE ha propuesto una solución consensuada en base a 2 pilares:

(i) El Pilar I - "Enfoque Unificado", que se centra en establecer un nuevo criterio de sujeción o nexo — ¿dónde deben

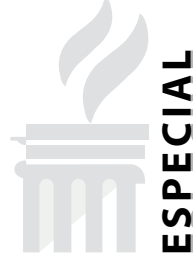


pagarse los impuestos? - y de asignación de utilidades - ¿cuánto beneficio asignar a cada país que conforma el modelo de negocio?-. En otras palabras, el derecho que tiene un país de gravar las utilidades de determinada empresa no estará ligado a la noción de presencia física en esa jurisdicción. Por el contrario, se intenta dotar al país donde está localizado el mercado o los usuarios finales, con la potestad de gravar parte de la renta del negocio digital más allá de que la compañía que provee esos servicios no tenga presencia física - a través de una entidad legal-en dicho mercado.

(ii) El Pilar II - "Propuesta GLoBE", que se enfoca en lograr que los negocios digitales internacionales sean gravados con un impuesto mínimo global con una tasa promedio de $15 \%$ - que desincentive la derivación de utilidades hacia jurisdicciones o regímenes fiscales preferentes. El objetivo aquí es desarticular las estructuras de planeamiento internacional bajo la utilización de derechos de propiedad intelectual hacia jurisdicciones de baja o nula tributación o la utilización abusiva de convenios para evitar la doble imposición o régimen fiscales particulares.

Si bien en octubre de 2020 la OCDE renovó su compromiso de alcanzar un acuerdo global a mediados de $2021^{11}$, a la fecha todavía no se ha conseguido consenso en implementar estas alternativas, debido a que los principales países tecnológicamente avanzados no están interesados en que se les aplique gravamen directo a las rentas que obtienen las EMNT residentes en dichos países.

2. Enfoque multilateral: Modelo Unión Europea - UE.
La UE ha propuesto las siguientes alternativas —en marzo 2018-:

\subsection{Impuesto a los Servicios Digitales — ISD.}

Propuesta para que se establezca con carácter temporal —hasta que se implante una solución digital global- un ISD que grave con una tasa del $3 \%$ a los ingresos procedentes de la prestación de determinados servicios digitales caracterizados por la creación de valor por parte del usuario que constituye una contribución esencial para la empresa que realiza esa actividad y gracias a la cual puede obtener ingresos. No se requiere que el proveedor de tales servicios esté establecido físicamente en la jurisdicción donde se encuentran los usuarios y se crea el valor. Lo que está sujeto a tributación son los ingresos obtenidos de la monetización de la contribución del usuario y no su participación.

Se propone que el sujeto pasivo del ISD sea la entidad cuyo importe total de ingresos mundiales comunicado en el último ejercicio financiero completo del que se dispone de un estado financiero supera los 750 millones de euros, y además, el importe total de los ingresos imponibles que ha obtenido dentro de la UE durante ese ejercicio financiero supera los 50 millones de euros; ello independientemente de que estuviera establecida en un Estado miembro o en una jurisdicción no perteneciente a la UE.

El acreedor tributario del ISD sería el Estado o Estados miembros donde están establecidos los usuarios, independientemente de que estos últimos hayan contribuido o no pecuniariamente a la generación de la renta de la empresa. Sin embargo, para su implementación se requiere de la cooperación administrativa, es decir, del intercambio de información entre los Estados miembros, necesaria para la identificación de los sujetos pasivos y la presentación de las declaraciones del ISD, así como para la transferencia de fondos correspondientes a los pagos de

11. Documento disponible en: https://www.oecd.org/tax/beps/cover-statement-by-the-oecd-g20-inclusive-framework-on-beps-on-the-reports-on-the-blueprints-of-pillar-one-and-pillar-two-october-2020.pdf. 
ISD por el Estado miembro de identificación a los demás Estados miembros en el que se adeude el impuesto.

\subsection{Presencia Digital Significativa.}

Propuesta similar a la de OCDE a fin que se establezca un nexo imponible para las empresas digitales que operan internacionalmente en caso de presencia comercial no física sino "presencia digital significativa" y se aplica a las entidades, independientemente del lugar en el que tengan su domicilio fiscal a efectos del impuesto sobre sociedades, si la actividad ejercida a través de la misma consiste total o parcialmente en la prestación de servicios digitales a través de una interfaz digital y siempre que la proporción de los ingresos totales obtenidos en ese periodo impositivo y resultante de la prestación de los servicios digitales a usuarios situados en dicho Estado miembro durante el mismo periodo impositivo sea superior a 7 millones de euros, y el número de usuarios de uno o más de los servicios digitales que estén situados en ese Estado miembro en dicho periodo impositivo sea superior a 100,000, y el número de contratos entre empresas para la prestación de tales servicios digitales que suscriban en ese periodo impositivo los usuarios situados en dicho Estado miembro sea superior a 3,000.

A la fecha tampoco se ha conseguido unanimidad entre los países miembros de la UE a fin de implementar estas propuestas, pues Irlanda, Dinamarca, Finlandia y Suecia se opusieron a dicha propuesta. Incluso son diez países miembros de la UE que han adoptado medidas unilaterales, por razones estrictamente recaudatorias.

\section{Respuesta unilateral de varios países.}

Ante la falta de consenso internacional para aceptar cualquiera de los modelos multilaterales antes expuestos, algunos países como Israel, Francia, Reino Unido, Austria, Hungría, Repúbli- ca Checa, Bélgica, España, Italia, Malasia, Turquía, India - publicidad-y otros, han optado por establecer de manera soberana y unilateral en sus legislaciones internas, un Impuesto único a los Servicios Digitales —Digital Services Tax, en inglés-, que grava con una alícuota de $3 \%$ - en promedio - a los ingresos que obtengan las EMNT provenientes de sus servicios digitales que presten en el país, a fin de resolver el problema de la falta de tributación en la jurisdicción donde se produce el consumo de los bienes o servicios.

Estas medidas unilaterales han provocado conflictos con los países donde se localiza, en última instancia, el capital de las EMNT, fundamentalmente, EE.UU. o China, siendo que países como Francia e Italia tuvieron que retroceder en la implementación de dicho impuesto ${ }^{12}$ ante amenazas de incremento de aranceles aplicables a sus productos.

Cabe indicar que en América Latina, países como Perú, Chile, México y Uruguay han recurrido a extender unilateralmente el concepto de Fuente de la renta, para incluir el uso de mercado como fuente de la renta, que le confiere jurisdicción sobre el Impuesto a la Renta al país donde se encuentra domiciliado el destinatario, cliente o usuario del servicio digital, independientemente donde se encuentre ubicado el proveedor o su Servidor, debiendo pagarse vía retención por la empresa domiciliada usuaria de los servicios digitales, quien en la práctica, asume dicho impuesto.

Dado que resulta difícil cobrarle el impuesto vía retención a las personas naturales, se está evaluando trasladarle la tarea de retención del impuesto a la renta a un tercero que por su ubicación, colabore con el Estado - podrían ser los bancos, entidades financieras que retengan el dinero, etc.-, aunque en la práctica, quien asumiría el traslado de dicho impuesto sería el consumidor, por la vía del aumento del precio de los servicios. 
Estas medidas unilaterales de retención en la fuente todavía están en evaluación, pues en tiempos de pandemia supone el encarecimiento del precio de los servicios digitales, y porque además generan efectos no deseables como son: 1) La doble o múltiple tributación, los conflictos del ejercicio de la potestad tributaria de uno o más Estados; 2) El incremento de costos de transacción de las empresas perceptoras o retenedoras del impuesto; 3) Dificultades operativas de los operadores para identificar las transacciones gravadas o no con el impuesto; entre otras.

Si bien la solución multilateral de fiscalidad directa a la economía digital sería lo más conveniente, a la fecha todavía no ha conseguido consenso en implementar esta propuesta de solución, por lo que se prevée que los demás países sigan adoptando medidas unilaterales por razones recaudatorias. 